\title{
A Rapidly Growing Transparent Braces Trend in Orthodontics: Clear Aligners- A Literature Review
}

\author{
Sharath Kumar Shetty ${ }^{1}$, Vijayananda K Madhur ${ }^{2}$, Eva Emelya Jose ${ }^{3 *}$, Mahesh Kumar Y ${ }^{4}$
}

\begin{abstract}
${ }^{1}$ Professor \& HOD, Department of Orthodontics and Dentofacial Orthopaedics, K. V. G. Dental College and Hospital, Sullia, Karnataka, India
${ }^{2}$ Reader, Department of Orthodontics and Dentofacial Orthopaedics, K. V. G. Dental College and Hospital, Sullia, Karnataka, India

${ }^{3}$ Post Graduate Student, Department of Orthodontics and Dentofacial Orthopaedics, K. V. G. Dental College and Hospital, Sullia, Karnataka, India

${ }^{4}$ Professor, Department of Orthodontics and Dentofacial Orthopaedics, K. V. G. Dental College and Hospital, Sullia, Karnataka, India
\end{abstract}

DOI: $10.36347 /$ sjds.2020.v07i12.008

| Received: 12.12.2020 | Accepted: 26.12.2020 | Published: 30.12.2020

*Corresponding author: Eva Emelya Jose

Abstract

Review Article

In recent years, increasing numbers of adult patients have sought orthodontic treatment 1 and expressed a desire for esthetic and comfortable alternatives to conventional fixed appliances. The transparency of the clear aligner enhances its esthetic appeal for those adult patients who are averse to wearing conventional labial fixed orthodontic appliances. Orthodontists should gain significant clinical experience in the treatment of mild malocclusions before attempting to treat more complex cases. One needs to understand that aligners are only an appliance, and the technique for working with it is continually being developed and honed. Refinement, adjustment at each appointment, and rebooting are all a part of the technique, and everything depends on the orthodontist's skill just as with patient with any fixed appliance.

Keywords: Orthodontics; Clear aligner; Invisible orthodontics; Invisalign®.

Copyright $\odot 2020$ The Author(s): This is an open-access article distributed under the terms of the Creative Commons Attribution 4.0 International License (CC BY-NC 4.0) which permits unrestricted use, distribution, and reproduction in any medium for non-commercial use provided the original author and source are credited.

\section{INTRODUCTION}

The increase in the number of adult orthodontic patients has prompted an upsurge in the demand for esthetic and comfortable alternatives to conventional fixed appliances [1-4]. Clear aligners that satisfy this demand are also prone to rapid technological improvements in aligner materials and production techniques [1]. Developments in clear aligner technologies have increased the number and complexity of cases treated with this method [5]. Clear aligners provide an esthetic and comfortable treatment experience, facilitate oral hygiene, cause less pain as compared to fixed orthodontic appliances, reduce the number and duration of appointments, and require less emergency visits [6, 7]. However, the expense in production, dependency on patient cooperation, and the inability to treat certain malocclusions limit the usage of clear aligners [1, 3, 5, 8-11].

\section{HISTORY}

The theory of using an aligner to straighten teeth was first postulated in the 1940s when Kesling produced a tooth positioning appliance to refine the final stages of orthodontic treatment [12]. This positioned was a piece of pliable rubber manufactured from a laboratory wax up of the teeth in a class I occlusion [13]. This appliance allowed for minor tooth movements to be achieved while maintaining alignment of the remaining teeth in the arch. Tooth control was difficult, and only tipping of crowns was possible. Kesling foresaw that more ambitious tooth movements could be realized with a series of aligners, while recognizing the limitations of the technology available to him at the time: 'Major tooth movements could be accomplished with a series of positioners by changing the teeth on the set-up slightly as treatment progresses. At present, this type of treatment does not seem to be practical. It remains a possibility, however, and the technique for its practical application might be developed in the future' [12].

Thirty years later [14], introduced an 'Invisible Retainer,' which used Kesling's idea of pre-positioning teeth on a master study model. Like Kesling's appliance, the 'Invisible Retainer' could only produce minor tooth movements, again achieving its results through the tipping of crowns. In the early 90s, Sheridan described a technique of using clear aligners in conjunction with interproximal tooth reduction [15]. The principle of producing minor tooth movements with individual aligners had not changed. A new 'Kesling set-up' was required for every tooth movement, and therefore, a new impression was taken at almost every visit. This process demanded a large amount of clinical and laboratory time. 
Align technology released their InvisalignH system in 1999. It was the first orthodontic appliance to use computer- aided design (CAD) and computer-aided manufacturing (CAM). Instead of requiring a new impression for each tooth movement, this technology allows for multiple tooth set-ups to be created from a single impression [16]. The advent of this digital process removed the impracticality of previous aligner systems and made Kesling's concept a reality.

\section{INDICATIONS FOR INVISALIGN APPLIANCES}

- Crowding (mild) and mal-aligned problems (1-5 $\mathrm{mm}$ )

- $\quad$ Spacing problems (1-5 mm)

- Deep overbite (Class II division 2)

- Narrow arches that can be expanded without tipping the teeth too much.

- For absolute intrusion (1 or 2 teeth)

- Lower incisor extraction for severe crowding cases.

- $\quad$ To tip the molar distally [17]

\section{CONTRAINDICATION}

- Tip control

- Torque control

- Moderate to severe open and deep bite cases [21]

\section{ADVANTAGES}

- Trays are aesthetic and comfortable

- Absence of metal brackets or wires prevents any tissue laceration.

- The invisible nature allows patients to smile with greater confidence

- Improved oral hygiene than during conventional treatment

- Unlike traditional braces, the trays can be removed for brushing, flossing, and eating.

- Smaller dental appointments.

- Retention facilitated.

- Decreased occlusal abrasion from parafunctional habits during treatment.

- Less technique sensitive than lingual appliances.

- Treatment duration is decided at the start of the treatment

- Avoids extractions

- Less visits to the dentist

- Healthier periodontal tissue

- Less risk of enamel decalcification by avoiding brackets $[18,19]$.

\section{DISADVANTAGES}

- Lack of recalls makes it patient dependent treatment

- Patient motivation is required

- Removal of the appliance during consumption of hot food/ drinks is necessary as it increases the chances of deformation of the appliance.

- Even though treatment time is pre-determined, non compliance of the patient may cause difficulty in obtaining the results.
- Poor oral hygiene

- Loss of appliances can not only increase the treatment period but also increase the cost factor [20].

\section{TRANSIENT PROBLEMS WITH CLEAR APPLIANCES}

- It may initially irritate oral mucosa and tongue causing soreness.

- A temporary alteration of speech, with slight lisping.

- These immediate discomforts are transient and vanish within a few days, as the patient gets accustomed to the appliance [21].

\section{MATERIALS}

Various thermoplastic materials are currently used for the fabrication of clear aligners, including polyvinyl chloride, poly urethane (PU), polyethylene texepthalate (PET), and polyethylene terepthalate glycol (PETG) [22].

\section{GENERATION}

First Generation: Earliest form of these systems were solely reliant on the aligner to achieve their results. No auxillary elements were incorporated.

Second Generation: Makes use of attachments to improve tooth movements. Clinicians could request composite buttons to be placed on the teeth and could also start to use inter maxillary elastics.

Third Generation: Attachments are now place automatically by the manufacturer's software where extrusion, de-rotation and root movements are required. Indentations in the aligners are fabricated where root torque is needed [23].

\section{SMART TRACK MATERIAL}

Practitioners would not be able to achieve excellent clinical results with this advanced Invisalign system if the aligners comprised a typical off-the-shelf aligner material. Scientists at Align developed a clear multi-layer polymeric material with the performance characteristics necessary for excellent control of tooth movement and treatment overall. Among the criteria for appliance performance are clarity, load/ deflection rate, resilience and shape recovery, activation, insertion force, working range, force magnitude, patient comfort, and tooth/ aligner contact control. Smart Track material is vital to the use of sophisticated techniques like Smart-Force and Smart Stage. Smart Track is a proprietary highly elastic material. Conventional aligner materials undergo stress relaxation as their molecules rearrange and lose a substantial amount of force in the initial days of aligner wear. Smart Track maintains more constant force over the time the patient wears the aligners to elicit excellent biological response for orthodontic movement. The elasticity of the material achieves more tooth movement with each aligner than 
other aligner materials. The result is improved tracking throughout treatment. Smart Track material more precisely conforms to tooth morphology, attachments, and interproximal spaces, hence stabilizing the contacts between the aligner and teeth providing for better control of tooth movement throughout treatment ${ }^{24}$

\section{CONSIDERATIONS IN TREATMENT}

\section{PLANNING}

1. Biology of tooth movement should be kept in mind. For example, distalizing a tooth $10 \mathrm{~mm}$ may not be possible dentally without the proper anchorage reinforcements such as a temporary anchorage device.

2. Have orthodontic principles govern and dictate the movements. For example, distalizing an entire arch as a unit and seeing it displaced in the software is possible, however, again without extraoral forces, this is unlikely to happen

3. When setting up the final overjet, consider not leaving a tight overjet, to accommodate the thickness of the aligner in determining the tooth position. Align default currently is to leave the overjet at $0.5 \mathrm{~mm}$ to allow for the thickness of the aligner in the overjet position.

4. Optimized attachments and aligner features are placed based on software algorithms to apply the optimal forces needed in the direction needed for the programmed tooth movement. Consider not replacing them or removing them to experience their improved effectiveness on tooth movements.

5. If the treatment plan has IPR, consider not removing IPR unless there is the ability to procline or expand the teeth in the arch form and the periodontium is stable. Lever-age the superimposition tool in the software to see the amount of tooth movement from initial to final and superimposed over the grid can give you more precise measurements. For the most precise measurements, the tooth movement table provides changes for both the crown and root.

6. For A-P correction cases, anchorage control must be maintained with the use of elastics. Check for precisions cuts for Class II or Class III correction and leverage the tool in the software.

7. Remember to take into consideration the treatment time needed to correct the Class II or Class III even when a virtual elastic simulation or bite jump at the end of treatment is shown. The one-stage jump is a simulation of the A-P correction and the expectation is that the A-P correction is occurring with the use of elastics or in some instances with surgery. Understanding the virtual elastic simulation will become increasingly important as Align looks at future offerings in the A-P correction space.

8. Virtual simulations or bite jumps also occur in other dimensions such as vertical. Remember that these are virtual and keep orthodontic principles in mind when correcting an open bite for example.
Having the 3D model virtual jump close an open bite will require some form of tooth movement to remove interferences and facilitate the auto-rotation of the mandible.

9. Precision bite ramps do not extend beyond $3 \mathrm{~mm}$ and, therefore, will not be in occlusion with overjets more than $3 \mathrm{~mm}$. If larger bite ramps would be needed, consider placing bite ramps on the canines and then switching to Precision bite ramps when the overjet is $3 \mathrm{~mm}$ or less.

10. Leverage the occlusal contacts tool to look for premature contacts as well as to finalize the occlusion. In some instances, leaving heavy occlusal contacts may be desired such as in deepbite cases to overcorrect for posterior extrusion. Be certain to inform the technician the intent is to keep the heavy occlusal contacts, otherwise they will be removed. At the same time, it is important to check the occlusal contacts, identify any premature or heavy interference, and have a plan during or at the end of treatment to eliminate them, whether through equilibration or restorative procedures.

11. Overcorrection is prescribed by some clinicians to compensate for the lag of tooth movements accomplished in the aligners. Keep in mind especially when presenting to patients that with overcorrection the final position of the model is accentuated 27].

\section{RECENT INVISALIGN PROTOCOL IMPROVEMENTS}

Recently, numerous improvements have been introduced to the protocol for use of the Invisalign system [25]. These changes fall into the categories of anterior/posterior corrections, staging for interproximal reduction, staging for tooth movements, attachments, and staging of tooth movements.

\section{Anterior/Posterior (A/P) Corrections}

- Setups are designed to allow easier visualization of the anticipated treatment goal when incorporating interarch elastics in the treatment plan.

- Elastic wear is recommended from the start of treatment, continuing until the desired $\mathrm{A} / \mathrm{P}$ correction has been achieved.

- Setups will default to display A/P bite corrections incorporating the effects of interarch elastics.

- The effect of elastics is simulated as a one-stage anterior-posterior movement at the end of treatment [28].

- Fewer aligners are required when simultaneous staging is employed.

- Distalization staging may be requested in the special instructions of the treatment form or during ClinCheck Review.

Staging for Interproximal Reduction (IPR)

- The timing of IPR is automatically staged when there is better access to interproximal contacts. 
- IPR will be staged when there is not a significant overlap between teeth to avoid performing IPR on surfaces that may be damaged by instruments.

- Saving necessary IPR may be needed for Bolton's discrepancies are aligned to avoid removing enamel on an angle.

\section{Staging for Tooth Movements}

- Cases are staged to enable combination movements to occur simultaneously for each tooth.

- The tooth that needs to move the most (lead tooth) will determine the minimum number of stages required.

- All teeth move throughout the duration of treatment [28].

\section{Attachments}

- Attachments are now placed in the middle of the crown vs. $2 \mathrm{~mm}$ from the gingival margin.

- There are reduced rotational and extrusion values to trigger automated attachment placement.

- Rotational attachments are automatically sized in proportion to the clinical crown.

- One mm thick vertical rectangular attachments are used for rotations of round teeth or canines as well as translation of teeth adjacent to an extraction site.

- Use of $1 \mathrm{~mm}$ thick (buccal-lingual dimension) horizontal beveled rectangular attachments is standard on premolars for retention of aligners during intrusive movements such as leveling the lower curve of Spee in deep overbite, for extrusions, and for control of the tooth long axis during torquing movements [28].

\section{Staging of Tooth Movements}

- Linear and rotational velocities of teeth are tracked separately.

- The minimal number of treatment stages is determined via the lead tooth based on its rotational or linear maximum velocity.

- Slower rotations are staged in treatment.

- Movements of all teeth are simultaneous [28].

\section{CONCLUSION}

The Invisalign System has opened up a new area of adult orthodontics, serving patients who may not want conventional fixed appliances or for whom traditional removable appliances may be unsuccessful. Educating patients on the advantages and disadvantages of clear aligner therapy or clear braces significantly depends on patient's expectations and compliance. They need to consistently wear the aligners 22 to 23 hours per day and only remove them to eat. It is essential to continuously motivate each patient during treatment to properly wear aligners to avail benefits of the treatment, ensure patient compliance as well as patient self discipline.
The use of the Invisalign appliance in combination with fixed appliances has been explored to reduce the time needed to wear fixed appliances, but may result in considerably higher professional fees overall. Conversely, the Invisalign appliance can provide an excellent esthetic during treatment, ease of use, comfort of wear, and superior oral hygiene.

\section{Conflict of interest: None}

\section{REFERENCE}

1. Weir T. Clear Aligners in Orthodontic Treatment. Aus Dent J. 2017; 62:58-62. doi: 10.1111/adj.12480.

2. Karkhanechi M, Chow D, Sipkin J, David S, Boylan R, Norman R, et al. Periodontal Status of adult patients treated with fixed buccal appliances and removable aligners over one year of active Orthodontic Orthod. 2013;83:146-51.

Theraphy. Angle

3. Melkos AB. Advances in digital technology and orthodontics: a reference to the Invisalign method. Med Sci Monit. 2005;11:39-42.

4. Malik $\mathrm{OH}$, McMullin A, Waring DT. Invisible orthodontics part 1: invisalign. Dent Update. 2013; 40:203-4. 207-10, 213-5.

5. Graber TM. Orthodontics: Current Principles and Techniques. In: Paquette D, Colville C, Wheeler $\mathrm{T}$, editors. Clear Aligner Treatment. St Louis: Mosby; 2012. pp. 778-811.

6. Fujiyama K, Honjo T, Suzuki M, Matsuoka S, Deguchi T. Analysis of pain level in cases treated with Invisalign aligner: comparison with fixed edgewise appliance therapy. Prog Orthod. 2014;15:64.

7. Miller KB, McGorray SP, Womack R, Quintero JC, Perelmuter M, Gibson J, Dolan TA, Wheeler TT. A comparison of treatment impacts between Invisalign aligner and fixed appliance therapy during the first week of treatment. American Journal of Orthodontics and Dentofacial Orthopedics. 2007 Mar 1;131(3):302-e1.

8. Proffit WR, Fields H, Sarver D. Contemporary Orthodontics. 5th ed. St. Louis: Mosby; 2013. pp. 355-7.

9. Drake C, McGorray S, Dolce C, Nair M, Wheeler T. Orthodontic Tooth Movement with Clear Aligners. ISRN Dent. 2012.

10. Ponitz RJ. Invisible retainers. Am J Orthod. 1971;59:266-72.

11. Nahoum HI. Forces and moments generated by removable thermoplastic aligners. American Journal of Orthodontics and Dentofacial Orthopedics. 2014 Nov 1;146(5):545-6.

12. Kesling HD. Coordinating the predetermined pattern and tooth positioner with conventional treatment. Am J Orthod Oral Surg. 1946; 32: 285 293.

13. Phan $X$ and Ling PH. Clinical limitations of Invisalign. J Can Dent Assoc 2007;73: 263-266. 
14. Ponitz RJ. Invisible retainers. Am J Orthod. 1971; 59: 266-272.

15. Sheridan JJ, LeDoux $\mathrm{W}$ and McMinn R. Essix retainers: fabrication and supervision for permanent retention. J Clin Orthod. 1993; 27: 3745.

16. Hajeer MY, Millett DT, Ayoub AF and Siebert JP. Applications of 3D imaging in orthodontics: Part II. J Orthod. 2004; 31: 154-162.

17. Murthy VS, Vijay. Orthodontics without braces andwires!! A New Paradigm. Indian Journal of Dental Advancements. 2011; 3(2):508-11.

18. Mehta F. Mehta S. Aligners: the rapidly growing trend in orthodontics around the world. Indian $\mathbf{J}$ Basic Applied Med Res. 2014;3(4): 402-9.

19. Sadri I. An argument for clear aligners and clearbraces.

20. Muggiano F, Quaranta A. The Aesthetic Alternativein Orthodontics with Sequential Aligners: TheInvisalign System. Webmed Central Orthodontics. 2013;4(10):1-6.

21. Hirani S, Patel U, Patel N. Invisible OrthodonticsA Review. J Dent Med Sci. 2016; 15(6):56-62.
22. Chen-Lu Liu, Wen-Tian Sun. Colour Stabilities of three types of orthodontic clear aligners exposed to staining agents. Int J Oral Sciences. 2016; $8(4): 246-53$.

23. Joe Hennessy and Ebratim A. Al-Awadhi. Clear aligners generations and orthodontic tooth movement. J Orthod. 2016;43(1):68-76.

24. Morton J, Derakhshan M, Kaza S, Li C. Design of the Invisalign system performance Sem in Orthod. 2017; 23:3-11.

25. Boyd RL. Complex orthodontic treatment using a new protocol for the Invisalign appliance. J Clin Orthod. 2007; 41:525-47.

26. Miller RJ, Duong TT, Derackhshan M. Lower incisor extraction treatment with the Invisalign system. J Clin Orthod. 2002; 36(2):95-102.9

27. Morton J, Derakhshan M, Kaza S, Li C. Design of the Invisalign system performance. Sem in Orthod. 2017; 23:3-11.

28. Boyd RL. Esthetic orthodontic treatment using the invisalign appliance for moderate to complex malocclusions. Journal of dental education. 2008 Aug; 72(8):948-67. 